\title{
Brain-Gut Molding Aberrant Development Trajectory in Autistic Children
}

\author{
Edwin Chau Leung YU* \\ Director, InteMed HK; Hong Kong
}

Submission: February 21, 2019; Published: March 08, 2019

*Corresponding author: Edwin Chau Leung YU, Director, InteMed HK, Shan Tung Street, Mongkok, Kowloon, Hong Kong

Keywords: Autism spectrum disorders; Brain-Gut; Hypoplasia

\section{Review}

Autism spectrum disorders (ASDs) are neurodevelopmental disorders. Many divergent pathogenetic views are currently present. Atypicality's of structural and functional development of the brain have been used to explain ASD, but yet inconclusive. A recent paper tried to demonstrate these prenatally and perinatally (hypoplasia of the pons just after neural tube closure; and a deficient GABA developmental switch in the perinatal period) [1]. Nevertheless, these specific prenatal and perinatal causes are expected to be infrequent. Yet ASD is common, affecting up to $2.25 \%$ of children [2,3], even prevalent with parents highly educated. Pathogenesis is also sought for genetic and environmental factors yet ASD etiology remains unknown [4].

The difficulty to find the etiology throughout the years may imply complex causes that work together in bringing forth an autistic child. And these factors may sequentially perpetuate the problem. Assembly of the developing nervous system for a final functional neural circuitry is dependent on a series of temporally regulated developmental processes.

\section{Explaining Autism Neurologically}

Development follows senses and stimulation. Thus, as past socio-medical approach to ASD became more sophisticated, the social motivation theories were used to explain the ASD manifestations of stereotypical behavior, communication, and social interaction deficits. The hypothesis suggests that early neurobiological difference in response to social input could lead to weakened social motivation, and in time motivation for restricted interests increases [5].

Carried these up the brain, cognitive models developed. These include domain-specific models elaborating primary deficit in social cognition, and domain general models elaborating primary deficit in nonsocial or domain-general processing. The disrupted cerebral connectivity hypothesis postulates that its clinical symptoms originate from deficiencies in the way the brain coordinates and synchronizes activity amongst different brains regions [6].

\section{More understanding starting from Gut Microbiota}

The gut microbial community is dynamic during the first 3 years of life and then stabilizes to an adult-like state [7]. Gut bacteria influence the central processes through their ability to synthesize neurotransmitters including gamma-aminobutyric acid, noradrenaline, and dopamine, modulate activation of the immune system, and produce metabolites, such as short-chain fatty acids that possess neuroactive properties [8]. Research has demonstrated the association between gut microbiota and early cognition in human infants. Fecal microbial community diversity in infants affects later Mullen score (scale of early learning), visual reception scale, and expressive language scale at two years of age [9].

Early life stress during this critical period can induce alterations in many body systems. Time windows of microbial community might be critical in shaping the brain function and have long-lasting effects on behaviors. Perturbations in the delicate synergetic host- microbiota relationship may have serious consequences and lead to brain, digestive, and metabolic disorders [10].

The microbiota-gut-brain axis exerts a profound influence on key brain processes, such as neuroinflammation, activation of the stress axes, neurotransmission, and neurogenesis, in addition to modulating complex behaviors, such as sociability and anxiety [11]. 
Neuroinflammation-related brain injuries are associated with [12] and cytokine imbalance is involved in ASDs [13]. Neuroinflammation contributes to a significant subset of ASDs [14].

\section{Gut - more than just Microbiota}

During rapid volume growth of cortical gray matter in the first 2 years of life, certain brain regions, notably those around information processing sensory modalities, develop faster. These regions include the inferior frontal gyrus and angular gyrus, cortical regions involved with language, the fusiform gyrus, involved with face recognition and color processing and the inferior temporal gyrus, involved with higher-order visual processing, including shape and faces. Oddly, the insula is also one of the most rapidly growing regions. In fact, the insula is involved with awareness of interoceptive or visceral sensations, pain, body movement, emotions, vocalizations, and perhaps even consciousness [15-16]. Visceral sensations develop as the enteric nervous system (ENS) develops during interactions of the neural crest-derived precursors (mostly vagal neural crest cells [17]) with the enteric microenvironment.

Luminal stimuli activate mucosal enteroendocrine cells and initiate peristaltic and secretory reflexes [18]. Besides the mainframe brain, some called ENS the 'little brain'. The ENS is a division of the autonomic system put in close apposition to effector systems it controls; enterohormones also co-working.

\section{Memory also related to Gut feelings}

Hippocampal volume rapidly increases in the first two years of life [19], though less in the first year compared with the other subcortical structures. With more mobility of the child in the second year of life, hippocampus becomes one of the faster growing structures, as it supports the acquisition of episodic memory [20] as well as spatial working memory and path integration abilities [21].

The hippocampus is activated by enteric signals through the vagus nerve between the intestinal tract and the brain [22]. The hippocampus is linked with learning and memory control and with feeding behavior [23]. Vagus nerve stimulation enhances memory [24], facilitates hippocampal neurogenesis, increases hippocampal expression of brain-derived neurotrophic factor [25] and induce neuronal plasticity [26]. Neurogenesis with new neurons continues in the hippocampus to play an important role in learning and memory and responses to stress, even till adulthood [27].

Dopamine dysfunctions have been reported in ASD, and autistic-like behavior could arise from dopamine dysfunctions in midbrain dopaminergic modulatory systems affecting social motivation and goal-directed motor behavior [28]. Dopamine affects plasticity, synaptic transmission and the network activity in the hippocampal circuitry for memory [27]. Findings suggest that while memory representations are processed and activated by the hippocampus in both ASD and controls during successful retrieval, these are not searched for, transferred, or monitored in an efficient way during episodic memory retrieval as a result of widespread disrupted connectivity [29].

\section{Aberrant development Trajectory in Autistic children}

The developmental trajectory before the preschool years is determined by a combination of internal biologic variables and environmental influences [30]. It depends on the performance level at the onset and the rate of change and direction of deviation. Memory deficits in ASD driven by retrieval-related impairments reduce their probability of recollection success [29]. As it biases the individual's social motivation in a more or less fixed environmental structure, the individual conforms with a selfconcept and self-worth in a fairly fixed trajectory pertaining to himself. Though parenting style and learning environment can have significant effects, the individual developmental trend of mental functioning at a different level and discrimination tends to be fixed. This could lead to diminished social motivation and increased activation to stimuli associated with their restricted interest and thereby the typical ASD manifestations.

Developmentally, visceral sensations and the enteric nervous system develop together. Stimulation with external or internal (visceral) information is important for developing functional networks and refining synaptic plasticity. A study noted infants with sleep restlessness starting after 3 months old can recover with peaceful sleep by 3-5 courses every 7-10 days of chlorpheniramine $1 \mathrm{mg}$ and duphalac $2.5 \mathrm{ml}$ daily for 3 days [31]. During early development, time windows shaped by enteric microbial assembly and cortical neuro connectivity may shift developmental trajectories for brain function and behaviors.

ASDs having neuro-inflammation with deviations in gut microbiota, and problems in acquisition of memory and path integration abilities related to affected hippocampus neurogenesis, could set up an aberrant developmental trajectory.

Successful treatment of ASD children with herbs, senna and chlorpheniramine, by improving intestinal transit time, relieving inflammation and improving sleep, noted first and early recovery of the enteric system (appetite, speed of finishing feeds) [32]. Subsequently there was improvement in sleep restlessness, concentration, temper, and school acceptance. Finally, the children had restoration of normal school work and social interaction. Together with the restoration of the internal environment and the management of abnormal processes, ASD children could recover with treatment and restore their useful activities and normal development trajectories.

\section{Aberrant development Trajectory perpetuated in Au-} tism

Aberrant connectivity may lead to structural demonstrable differences in many brain areas, especially for developmental process involved in response to rewarding social input, which in turn may lead to the diminished social motivation. Apart 
from the aberrant connectivity, other issues in ASDs may perpetuate the problem. Brain development including brain cell proliferation, apoptosis, myelination, neurogenesis, maturation and differentiation depend on nutrition and immune development from the gut [33]. The temporal series of adaptive mechanisms would pave the developing nervous system for a final functional neural circuitry.

The treatment report, after enteric restoration, noted that improvement of sleep precedes improvement of other problems [32]. ASD tends to be associated with difficulty in falling asleep, wake up in the night frequently and a low frequency of saccadic eye movement during REM sleep [34]. Slow wave sleep is also shortened in ASD, and sleeping time, particularly the proportion of REM sleep, is reduced [35]. Cerebral plasticity has an important relationship with sleep [36].

Inadequate or poor sleep may foster lasting neural changes as well as changes in functional connectivity after perceptual, motor, or emotional learning tasks [37]. Hippocampus and the ventral striatum are activated during NREM sleep [38], and this may help subsequent performance improvement [39] and consolidate associative memory-reward information [40].

Noted in rats, REM sleep deprivation impairs hippocampal neurogenesis and related memory formation [41]. Emotional offrelevance and motivational biases may misdirect this overnight consolidation of declarative memory and skill learning [42].

Poor sleep, probably disturbed ENS visceral sensations, memory deviations, emotional off- relevance and motivational biases, associative memory-reward value-system, and neuro- inflammation as well as habituated responses to environment make up a set of factors in ASDs that perpetuate the aberrant development trajectory. It deters useful interactions with the environment, leads to the diminished social motivation, biased social skills and restricted interests. Whether due to a circuit dysfunction in reward and motivation, autistic brains would fail to register social experiences as rewarding, further reducing social interactions and social abilities, ultimately leading to heterogeneous social deficits with this "aberrant development trajectory" [32].

\section{Conclusion}

ASD may arise from deviated hippocampus memory processing related to brain-gut dysregulation. There is a critical window in early life, when the cerebral cortical networks from neuroconnectivity is adapting to changes, that microbial colonization can influence neurogenesis, including that in the hippocampus. ASDs are associated with neuro- inflammation, and alterations in the gut microbiota can change the developmental trajectory of brain function and behavior.

The vagus nerve of the intestine, with which the enteric microenvironment inputs related memory to hippocampus, will affect neural development for hippocampus. ASD children have memory retrieval-related impairments that reduce the probability of recollection success. These aberrant memory processes, resulting in restricted interest and diminished social motivation. It explains how ASD children have stereotypic interest and behavior, their characteristic communication and social interaction deficits, as well as the presence of atypical mixed picture of neural connectivity.

The developmental trajectory of ASD with perpetuating brain-gut dysregulation, working memory and path integration deviations, poor restoration from sleep all result in related brain function and behavior deviating from normal. A course of Chinese and western medicines has been successful to restore intestinal function, improve sleep, restore normal school work and social interaction. Treatment that normalizes microbiota relieving neuro-inflammation and management of the enteric system can be useful in maintaining signals to the hippocampus and brain for neurogenesis. Together, with improving sleep, restoration of the internal environment and the management of abnormal processes, it is very likely that ASD can be treated to restore their useful activities and normal development trajectories of life.

\section{References}

1. Inui T, Kumagaya S, Myowa-Yamakoshi M (2017) Neurodevelopmental Hypothesis about the Etiology of Autism Spectrum Disorders. Front Hum Neurosci 11: 354.

2. American Psychiatric Association (2013) Diagnostic and Statistical Manual of Mental Disorders. 6th Edn. American Psychiatric Press, Washington DC, USA.

3. Zablotsky B, Black LI, Maenner MJ, Schieve LA, Blumberg SJ (2014) Estimated prevalence of autism and other developmental disabilities following questionnaire changes in the 2014 national health interview survey. Natl Health Stat Report 87: 1-20.

4. de la Torre-Ubieta L, Won H, Stein JL, Geschwind DH (2016) Advancing the understanding of autism disease mechanisms through genetics. Nat Med 22(4): 345-361.

5. Clements CC, Zoltowski AR, Yankowitz LD, Yerys BE, Schultz RT, et al. (2018) Evaluation of the Social Motivation Hypothesis of AutismA Systematic Review and Meta- analysis. JAMA Psychiatry 75(8): 797808.

6. Vasa RA, Mostofsky SH, Ewen JB (2016) The Disrupted Connectivity Hypothesis of Autism Spectrum Disorders: Time for the Next Phase in Research. Biol Psychiatry Cogn Neurosci Neuroimaging 1(3): 245-252.

7. Yassour M, Vatanen T, Siljander H, Hämäläinen AM, Härkönen T, et al. (2016) Natural history of the infant gut microbiome and impact of antibiotic treatment on bacterial strain diversity and stability. Sci Transl Med 8(343): 343ra81.

8. Sherwin E, Sandhu KV, Dinan TG, Cryan JF (2016) May the force be with you: the light and dark sides of the microbiota-gut-brain axis in neuropsychiatry. CNS Drugs 30(11): 1019-1041.

9. Carlson AL, Xia K, Azcarate-Peril MA, Goldman BD, Ahn M, et al. (2018) Infant Gut Microbiome Associated With Cognitive Development. Biol Psychiatry 83(2): 148-159.

10. Grenham S, Clarke G, Cryan JF, Dinan TG (2011) Brain-gut-microbe communication in health and disease. Front Physiol 2: 94.

11. Cryan JF, Dinan TG (2012) Mind-altering microorganisms: the impact of the gut microbiota on brain and behaviour. Nat Rev Neurosci 13(10): 701-712. 
12. Jin C, Londono I, Mallard C, Lodygensky GA (2015) New means to assess neonatal inflammatory brain injury. J Neuroinflammation 12: 180.

13. Siniscalco D, Schultz S, Brigida AL, Antonucci N (2018) Inflammation and Neuro- Immune Dysregulations in Autism Spectrum Disorders. Pharmaceuticals 11(2): E56.

14. Bilbo SD, Block CL, Bolton JL, Hanamsagar R, Tran PK (2018) Beyond infection- Maternal immune activation by environmental factors, microglial development, and relevance for autism spectrum disorders. Exp Neurol 299(Pt A): 241-251.

15. Nagai M, Kishi K, Kato S (2007) Insular cortex and neuropsychiatric disorders: a review of recent literature. Eur Psychiatry 22(6): 387-394.

16. Craig AD (2009) How do you feel--now? The anterior insula and human awareness. Nat Rev Neurosci 10(1): 59-70.

17. Uesaka T, Young HM, Pachnis V, Enomoto H (2016) Development of the intrinsic and extrinsic innervation of the gut. Dev Biol 417(2): 158-167.

18. Gershon MD (2009) Enteric Nervous System Development. In: Binder MD, Hirokawa N, et al. (Eds.) Encyclopedia of Neuroscience. Springer Verlag, Heidelberg, Germany.

19. Gilmore JH, Shi F, Woolson SL, Knickmeyer RC, Short SJ, et al. (2012) Longitudinal Development of Cortical and Subcortical Gray Matter from Birth to 2 Years. Cerebral Cortex 22(11): 2478-2485.

20. Tustin K, Hayne H (2010) Defining the boundary: age related changes in childhood amnesia. Dev Psychol 46(5): 1049-1061.

21. Wolbers T, Wiener JM, Mallot HA, Buchel C (2007) Differential recruitment of the hippocampus, medial prefrontal cortex, and the human motion complex during path integration in humans. J Neurosci 27(35): 9408-9416

22. Suarez AN, Hsu TM, Liu CM, Noble EE, Cortella AM, et al. (2018) Gut vagal sensory signaling regulates hippocampus function through multiorder pathways. Nature Comm 9(1): 2181.

23. Kanoski SE, Grill HJ (2017) Hippocampus contributions to food intake control: mnemonic, neuroanatomical, and endocrine mechanisms. Biol Psychiatry 81(9): 748-756.

24. Clark KB, Naritoku DK, Smith DC, Browning RA, Jensen RA (1999) Enhanced recognition memory following vagus nerve stimulation in human subjects. Nat Neurosci 2(1): 94-98.

25. Follesa P, Biggio F, Gorini G, Caria S, Talani G, et al. (2007) Vagus nerve stimulation increases norepinephrine concentration and the gene expression of BDNF and bFGF in the rat brain. Brain Res 1179: 28-34.

26. Biggio F, Biggio F, Gorini G, Caria S, Talani G, et al. (2009) Chronic vagus nerve stimulation induces neuronal plasticity in the rat hippocampus. Int J Neuropsychopharmacol 12(9): 1209-1221.
27. Snyder JS, Soumier A, Brewer M, Pickel J, Cameron HA (2011) Adult hippocampal neurogenesis buffers stress responses and depressive behaviour. Nature 476(7361): 458-461.

28. Pavăl D (2017) A Dopamine Hypothesis of Autism Spectrum Disorder. Dev Neurosci 39(5): 355-360.

29. Cooper RA, Richter FR, Bays PM, Plaisted-Grant KC, Baron-Cohen S, et al. (2017) Reduced Hippocampal Functional Connectivity During Episodic Memory Retrieval in Autism. Cerebral Cortex 27(2): 888-902.

30. Hamel SC, Pelphrey A (2009) In Developmental-Behavioral Pediatrics ( $4^{\text {th }}$ edn).

31. Yu ECL. Personal observation.

32. Yu ECL (2018) Neurodevelopment, Intestinal Function, and Autism. Neonat Pediatr Med 4(2): 166.

33. Fernandez AM, Torres-Aleman I (2012) The many faces of insulin-like peptide signalling in the brain. Nat Rev Neurosci 13(4): 225-239.

34. Limoges E, Mottron L, Bolduc C, Berthiaume C, Godboutet R (2005) Atypical sleep architecture and the autism phenotype. Brain 128(pt 5): 1049-1061.

35. Buckley AW, Rodriguez AJ, Jennison K, Buckley J, Thurm A, et al. (2010) Rapid eye movement sleep percentage in children with autism compared with children with developmental delay and typical development. Arch Pediatr Adolesc Med 164(11): 1032-1037.

36. Gorgoni M, D’Atri A, Lauri G, Rossini PM, Ferlazzo F, et al. (2013) Is Sleep Essential for Neural Plasticity in Humans, and How Does It Affect Motor and Cognitive Recovery? Neural Plasticity 2013:103949.

37. Payne JD, Kensinger EA (2010) Sleep leads to changes in the emotional memory trace: evidence from fMRI. J Cogn Neurosci 23(6): 1285-1297.

38. Nofzinger EA, Buysse DJ, Miewald JM, Meltzer CC, Price JC, et al. (2002) Human regional cerebral glucose metabolism during non-rapid eye movement sleep in relation to waking. Brain 125(pt 5):1105-1115.

39. Peigneux P, Laureys S, Fuchs S, Collette F, Perrin F, et al. (2004) Are spatial memories strengthened in the human hippocampus during slow wave sleep? Neuron 44(3): 535-545.

40. Lansink CS, Goltstein PM, Lankelma JV, McNaughton BL, Pennartz CM (2009) Hippocampus leads ventral striatum in replay of place-reward information. PLoS Biol 7:e1000173.

41. Meerlo P, Mistlberger RE, Jacobs BL, Heller HC, McGinty D (2009) New neurons in the adult brain: the role of sleep and consequences of sleep loss. Sleep Med Rev 13(3): 187-194.

42. Fischer S, Born J (2009) Anticipated reward enhances offline learning during sleep. J Exp Psychol Learn Mem Cogn 35(6): 1586-1593.

\section{Your next submission with Juniper Publishers will reach you the below assets}

- Quality Editorial service

- Swift Peer Review

- Reprints availability

- E-prints Service

- Manuscript Podcast for convenient understanding

- Global attainment for your research

- Manuscript accessibility in different formats

( Pdf, E-pub, Full Text, Audio)

- Unceasing customer service

\section{Track the below URL for one-step submission}

https://juniperpublishers.com/online-submission.php 